\title{
Analyse de la production écrite des apprenants du français comme L2
}

\author{
Kuok-Wa Chao ChaO
}

Escuela de Lenguas Modernas

Universidad de Costa Rica

\begin{abstract}
Résumé
Cet article présente les résultats obtenus de l'analyse de la production écrite de quatre groupes d'apprenants (troisième et sixième années) des écoles publiques bilingues français-espagnol Fernando Terán Valls et República Francesa par rapport aux aspects suivants : syntaxe, morphologie, lexique et orthographe. Il s'agit d'une étude transversale faite au mois d'octobre, 2013. Nous trouvons comme principales conclusions que l'interlangue de ces apprenants se ressemble beaucoup car nous avons des phénomènes d'interférences, de surgénéralisation et règles propres à l'interlangue.
\end{abstract}

Mots clés : français, interlangue, étude transversale, enfants, L2, production écrite, morphosyntaxe, lexique, orthographe

\section{Resumen}

Este artículo presenta los resultados obtenidos del análisis de la producción escrita de cuatro grupos de aprendices (tercero y sexto grados) de las escuelas bilingües públicas francés-español Fernando Terán Valls y República Francesa en cuanto a los siguientes aspectos: sintaxis, morfología, léxico y ortografía. Esta investigación de tipo transeccional se llevó a cabo durante el mes de octube del 2013. Las conclusiones principales que se obtuvieron con esta investigación son la semejanza de la interlengua de esos aprendices, ya que se encontraron fenómenos de interferencia, de sobregeneralización y reglas propias de la interlengua.

Palabras claves: francés, interlengua, estudio transeccional, niños, L2, producción escrita, morfosintaxis, léxico, ortografía 


\section{Introduction}

$\mathrm{L}$ e processus d'acquisition et d'apprentissage d'une langue seconde (L2) est très complexe car nous trouvons plusieurs facteurs qui interviennent, tels que l'interférence, la surgénéralisation, entre autres. Tous ces phénomènes sont présents dans l'interlangue des apprenants. Cette interlangue est le reflet d'une phase transitoire par laquelle tous les apprenants doivent passer pour pouvoir acquérir complètement leur L2.

Nous présentons dans cet article l'analyse de l'interlangue des productions écrites de quatre groupes d'apprenants appartenant aux écoles publiques bilingues. Nous analyserons d'abord les productions des apprenants de la troisième année; et puis, celles de la sixième année

\section{La théorie de l'interlangue}

Le concept d'interlangue a été traité par plusieurs auteurs (Corder, 1967, Selinker, 1972, Torras, 1994, Brou-Diallo, 2007, Fonseca, 2007, Khelef, 2008). Pour cette recherche, nous comprenons ce terme comme le système approximatif de connaissance de la langue cible que l'apprenant se construit mentalement sur la base des différences et des ressemblances qu'il observe entre la langue cible et la langue source, ainsi que sur la base de principes universels. Cette langue de l'apprenant se forme à partir des éléments auxquels il est confronté de la langue cible, sans pour autant qu'elle coïncide totalement avec cette langue cible car elle partage certains caractères de la L1 ou d'autres langues acquises par l'apprenant, certains caractères de la L2 et certains caractères propres à l'interlangue. C'est pour cela qu'elle est « hétérogène et naturellement instable puisqu'elle s'approche par degré de la L2, à mesure que l'appropriation de celle-ci se fait " (Monville-Burston et Kounouni, 2010, p. 1).

L’interlangue représente aussi les différentes étapes intermédiaires du système linguistique car elle représente la transition entre ce que l'apprenant sait déjà de la langue cible et ce qu'il n'a pas encore intériorisé.

\subsection{Les caractéristiques de l'inter- langue}

Différents auteurs (Torras, 1994, Galligani, 2003, Yun, 2009, Alexopoulou, 2010), l'interlangue parlent des caractéristiques de l'interlangue:

a. yle système de la langue cible. Les apprenants simplifient la langue cible parce qu'ils ont besoin de réduire la complexité des opérations mentales qu'ils doivent mettre en place. C'est pour cela que pendant les premières étapes du processus d'acquisition, les apprenants construisent leur discours à base des phrases simples en employant très peu de phrases complexes. Au niveau morphologique, les apprenants ont la tendance d'omettre les mots fonctionnels (verbes auxiliaires, prépositions, déterminants, etc.) ou les suffixes. Au niveau lexical, les apprenants peuvent recourir aux mots de leur L1 pour pouvoir communiquer.

b. La systématicité. L'interlangue est systématique. Cela veut dire qu'elle 
constitue en elle-même un système linguistique propre qui est régi par ses propres règles qui peuvent être différentes à celles de la langue cible ou de la L1 des apprenants.

c. Le dynamisme. L'interlangue est un système dynamique, c'est-àdire qu'elle change à mesure que les apprenants intègrent de nouvelles règles dans le système. Donc, chaque phase constitue en ellemême une compétence transitoire et toutes les phases représentent ce continuum d'interlangues.

d. La fossilisation. Elle peut être comprise comme la persistance d'habitudes articulatoires ou grammaticales de la L1, c'est-à-dire c'est lorsque l'apprenant ne continue plus à progresser pendant son processus d'acquisition de la langue cible, sinon ses formes linguistiques non correctes se stabilisent.

e. La perméabilité. Elle reflète un état transitoire car elle représente un constat d'incompétence en langue cible et un retour aux règles grammaticales de la langue maternelle pour une production peut-être compréhensible, mais grammaticalement inacceptable.

\subsection{Les processus constitutifs de l'interlangue}

D’après Azzam-Hannachi (2005), les différents processus pour aboutir à l'interlangue sont :

a. Transfert d'éléments de la L1. Elle consiste à utiliser des éléments de L1 quand on parle ou écrit une autre langue. b. Transfert d'enseignement. Elle consiste à appliquer une technique didactique non adéquate lors de l'enseignement d'une L2.

c. Stratégie d'apprentissage. Elle consiste à utiliser des techniques élaborées lors de l'acquisition de la L2.

d. Stratégies de communication. Elle représente l'emploi de techniques de compensation de déficits langagiers.

e. Surgénéralisation des règles de la langue cible. Elle consiste à appliquer de façon trop générale une règle découverte pour un ou plusieurs fondements langagiers

\section{Méthodologie}

Cette recherche a les caractéristiques d'une étude transversale car nous avons travaillé avec tous les groupes de la troisième et de la sixième années des écoles República Francesa et Fernando Terán Valls Lycée à un moment de leur processus d'acquisition et apprentissage du français comme L2.

Ce groupe d'apprenants est composé de 51 apprenants de la troisième année et de 53 apprenants de la sixième année. Les apprenants de la troisième année ont tous entre 8 et 9 ans et ceux de la sixième année ont tous entre 12 et 13 ans. Ils ont l'espagnol pour langue maternelle.

Le corpus de cette recherche est composé de productions écrites. Pour la troisième année, ils ont rédigé une seule composition de cinq lignes par rapport à la présentation personnelle. Pour la sixième année, ils ont rédigé deux compositions : une par rapport aux dernières vacances ; et pour l'autre, ils devaient écrire une lettre d'invitation. 
Toutes les productions écrites ont été recueillies pendant la première semaine du mois d'octobre 2013.

\section{Analyse des résultats}

Pour l'analyse des résultats, nous allons procéder d'abord à présenter ceux des apprenants de la troisième année ; et puis, ceux de la sixième année. Chaque année sera divisée par école.

Chaque partie sera composée de quatre aspects : syntaxe, morphologie, lexique et orthographe.

\subsection{L'analyse des productions écrites des apprenants de la troisième année}

Nous présenterons d'abord l'analyse des productions écrites de l'école República Francesa ; et puis, celle de l'école Fernando Terán Valls.

\subsubsection{Republica francesa}

\subsubsection{Aspect syntaxique}

En ce qui concerne l'aspect syntaxique, nous pouvons dire que les apprenants ont pu acquérir la règle de la structure de la phrase simple du français: sujet + verbe + complément, par exemple: j'ai 9 ans, j'aime les animaux, j'aime les chiens, *j'aime le ciclisme, il a 10 ans, elle a 11 ans.

Nous trouvons aussi quelques phrases avec la structure sujet + complément + verbe, à savoir je m'appelle..., ma sœur s'appelle Priscilla, mon frère s'appelle Juan.

Nous avons aussi des structures avec un verbe modal et un verbe infinitif, par exemple : j'aime manger, *j'aime aller à la pisine, *j'aime faire du velo.

Nous avons aussi des cas où les apprenants ne mettent pas de déterminants pour les groupes nominaux, par exemple :*jouer $\varnothing$ fotbal.

\subsubsection{Aspect morphologique}

Par rapport à l'aspect morphologique, nous voyons que les apprenants ont pu intérioriser les morphèmes du présent pour les verbes en -er de la première et de la troisième personne : je m'appelle, j'aime, je déteste, *je habite, ma sœur s'appelle, mon frère s'appelle, mon chien s'appelle. Ces apprenants ont aussi intériorisé quelques verbes irréguliers, par exemple : j'ai, il a, elle $\boldsymbol{a}$, ma famille est.

Cependant, nous avons des cas où les apprenants se servent du morphème -e ou des formes de la troisième personne singulier des verbes pour les autres personnes : *je est, *je a, *tu a, *ils a, *ils s'appelle. Nous trouvons aussi des cas où les apprenants utilisent la forme du verbe en infinitif au lieu de les conjuguer : *mon père se appeler, *ma famille se divertir, *je presenter, *je jouer. Nous avons un cas où l'apprenant se sert du morphème de la deuxième personne -es pour la première personne: *je aimes.

Quant aux déterminants, nous voyons que les apprenants ont pu acquérir les articles définis et indéfinis et quelques adjectifs possessifs : 
Tableau 1 : Les déterminants

\begin{tabular}{ll}
\hline \multicolumn{1}{c}{ Les articles } & \multicolumn{1}{c}{$\begin{array}{c}\text { Les adjectifs pos- } \\
\text { sessifs }\end{array}$} \\
\hline Le loisir & Mon père \\
Le chien & Mon frère \\
Le tigre & Mon chien \\
Le lion & Mon âge \\
Le cheval & *Mon ecole \\
Le chat & *Mon activite \\
*Le ciclisme & Ma mère \\
La T.V. & Ma sœur \\
La natation & Ma famille \\
*L'ecole & *Ma maisson \\
Les animaux & Mes animaux \\
Les chiens & $*$ Son aniversaire \\
Les ours & \\
Les piscines & \\
Un frere & \\
Une soeur & \\
*Un cha & \\
*Du velo & \\
*Des espaguetis & \\
Des chiens & \\
\hline
\end{tabular}

Nonobstant, nous avons des cas où les apprenants emploient le déterminant masculin pour déterminer les noms de genre féminin ou les noms au pluriel :

\section{Tableau 2 : L'utilisation des déterminants masculins}

\begin{tabular}{ll}
\hline Les articles & \multicolumn{1}{c}{$\begin{array}{c}\text { Les adjectifs } \\
\text { possessifs }\end{array}$} \\
\hline *Le picine & *Mon animaux \\
*Le jirafe & *Mon chats \\
*Le mer & $*$ Mon mere \\
*Un sœur & $*$ Mon maman \\
*Au piscine & $*$ Mon famille \\
& $*$ Mon meson \\
\hline
\end{tabular}

En ce qui concerne l'accord de l'adjectif et le nom ou le pronom, nous trouvons que les apprenants ne le font pas, à savoir *mon animaux préféré, *ma famille est gentil, *ma famille tre amusant.

Par rapport aux prépositions, nous voyons que dans l'interlangue des apprenants, nous trouvons l'utilisation de quelques-unes, telles que : *je habite à Ochomogo, *plus de activites. Cependant, nous apercevons que la préposition "dans " prend la fonction des autres prépositions, par exemple : *jouer dans le ordinateur, *dans ma famille il y a 5 personnes. Pour la première phrase, elle prend la fonction de la préposition "à " et pour l'autre phrase, elle prend la fonction de la préposition "chez".

Finalement, pour la contraction, nous voyons qu'il y a des apprenants qui n'appliquent pas la règle : *a les piscines.

\subsubsection{Aspect lexical}

Quant à l'aspect lexical, nous pouvons dire que ce groupe d'apprenants a un vocabulaire qui leur permet de faire une description simple d'une personne, une présentation, parler de la famille, de quelques animaux et de quelques activités: 
Tableau 3: Aspect lexical

\begin{tabular}{llll}
\hline Caractéristiques, qualités et couleurs & Actions & Lieux et activités & Autres \\
\hline Marron & Travailler & loisir & Famille \\
Blanc & Aimer & vélo & Frère \\
Noir & Détester & cyclisme & Père \\
Gros & Jouer & football & Mère \\
Content & Manger & marche & Soeur \\
Petit & Voyager & activité & animaux \\
Bon & Dessiner & ordinateur & chat \\
Gentil & Regarder & cinéma & chien \\
Grand & Colorier & école & crocodile \\
Amusant & & piscine & serpent \\
& & mer & tigre \\
& & théâtre & cheval \\
& & maison & vache \\
& & chambres & lion \\
& & & ours \\
\hline
\end{tabular}

Nous avons aussi des apprenants qui se servent de leur L1 pour parler de quelques aliments qu'ils mangent, par exemple: *lechuga, *espaguetis. Ils emploient aussi le lexème de leur L1 pour construire des mots de la L2: *comide, *delisie, *dibuyer. Nous avons aussi un cas de faux-amis avec le verbe "tenir" qui est utilisé avec le sens du verbe "avoir": *je tian.

\subsubsection{Aspect orthographique}

Par rapport à l'aspect orthographique, nous apercevons que la plupart des apprenants n'ont pas encore intériorisé les règles d'accentuation du français:

\section{Tableau 4: L'omission des accents}

\begin{tabular}{lll}
\hline \multicolumn{1}{c}{ Accent aigu } & Accent grave & Accent circonflexe \\
\hline *loisir prefere & $*$ aller a cinema & $*$ ages \\
$*$ velo & $*$ mere & \\
$*$ cinema & $*$ pere & \\
*animaux preferes & $*$ tres & \\
*je deteste & & \\
*ecole & \\
*je presente & \\
*activites & \\
\hline
\end{tabular}

Nous avons aussi des cas où les apprenants emploient l'accent aigu pour les mots qui portent un accent grave: *frére, *pére, *mére. En ce qui concerne l'élision, nous avons des cas où les apprenants appliquent la règle et des cas où ils ne la font pas: 
Tableau 5: L’élision

\begin{tabular}{ll}
\hline \multicolumn{1}{c}{ L'élision } & L'omission de l'élision \\
\hline Je m'appelle & *je aime \\
J'ai & *je me appelle \\
J'aime & *je ai \\
Ma soeur s'appelle & $\begin{array}{l}\text { *je appelle } \\
\text { *le ordinateur }\end{array}$ \\
\hline
\end{tabular}

Nous voyons aussi des cas où les apprenants remplacent les consonnes ou les voyelles, à savoir le remplacement de la consonne "g”" par “j”*jentis, *jirafe, de "j" par " $y$ " *yoli, de " $d$ " par " $t$ " *grant, de " $u$ " par " $a$ "* an frère, *an chien, de "i” par " $a$ " *lapan, de " $y$ " par " $i$ " *ciclisme, de "oi" par "u” *mua, de "oi" par "ou" *nouar et de "au” par "o" *otre.

Nous avons des cas d'omission des consonnes finales " $r$ ", " $s$ ", " $t$ ", " $l$ " ou de la voyelle " $e$ " en position finale, ou des doubles consonnes " $n$ ", " $s$ ", "r $r$, " $l$ ", ou de la consonne " $s$ " en position médiale:

Tableau 6 : L'omission de consonnes ou de voyelles

\begin{tabular}{ll}
\hline \multicolumn{1}{c}{ Consonnes } & \multicolumn{1}{c}{ Voyelles } \\
\hline *travailleØ & *tomatØ \\
*je deteste marcheØ & *frerØ \\
*jentiØs & *je m'appellØ \\
*groØ & *je presentØ \\
*petiØ & \\
*peroqueØ & \\
*treØ bon & \\
*aniversaire & \\
*persones & \\
*desine & \\
*paser & \\
*famile & \\
*picine &
\end{tabular}

Finalement, nous trouvons des cas d'omission du verbe « être » ou « avoir » :
Tableau 7 : L’omission « être » ou «avoir»

\begin{tabular}{|c|c|}
\hline être & avoir \\
\hline $\begin{array}{l}\text { *se Ø faire du velo } \\
* \text { se Ø jouer } \\
* \text { se Ø marron } \\
\text { *se Ø gro } \\
\text { *se Ø le chien } \\
\text { *se Ø tre bon } \\
\text { *se Ø grand } \\
\text { *ce Ø plus grand }\end{array}$ & *je Ø un frére \\
\hline
\end{tabular}

Comme synthèse, nous apercevons que ces apprenants ont pu intérioriser quelques règles dans leur interlangue, par exemple : la structure de base de la phrase du français, les morphèmes de la première et de la troisième personnes du singulier, l'apparition des quelques déterminants. Cependant, nous trouvons aussi des cas de surgénéralisation du déterminant masculin, l'omission de l'accord entre l'adjectif et le nom ou le pronom, la surgénéralisation de la forme de la troisième personne pour les autres personnes. Du point de vue de l'orthographe, nous pouvons dire qu'il y a surtout de l'interférence de la langue orale à la langue écrite comme les cas d'omission de consonnes ou de voyelles qui ne sont pas prononcées.

\subsubsection{Fernando Terán Valls}

\subsubsection{Aspect syntaxique}

Par rapport à l'aspect syntaxique, les apprenants ont pu acquérir la règle de la structure simple du français " sujet + verbe + complément ou sujet + verbe + attribut ", par exemple : j'ai 9 ans, il a 33 ans, *ma maman travaille dans un hotel, mon papa est gentil, c'est le lion. Nous 
trouvons aussi quelques structures " sujet + complément + verbe " dans l'interlangue de ce groupe d'apprenants : je m'appelle..., on frère s'appelle Geovanny, le chien s'appelle Bianca.

Nous avons aussi des cas où les apprenants n'ont pas encore intériorisé la place des éléments négatifs dans la phrase, par exemple : *jai ne pas $d u$ papa. Nous apercevons aussi des cas d'omission de déterminants pour déterminer un nom : *Carlos vais a la picine avec $\boldsymbol{\varnothing}$ amis. Finalement, nous trouvons des cas d'addition de la préposition " à " pour le futur proche : ${ }^{*}$ Carlos vais a manger. Probablement, pour la phrase précédente, nous assistons à un cas d'interférence de la L1 de l'apprenant.

\subsubsection{Aspect morphologique}

Quant à l'aspect morphologique, nous voyons que les apprenants ont pu intérioriser les morphèmes du présent pour les verbes en -er de la première et de la troisième personne : je m'appelle, j'aime, je dessine, j'habite, mon frère s'appelle, mon papa s'appelle, le chien s'appelle, mon papa travaille, ma maman travaille. Ils ont pu intérioriser quelques formes du présent des verbes " être ", " aller " et " avoir " dans leur interlangue : j'ai, Carlos va, mon papa est, il $\boldsymbol{a}$, elle $\boldsymbol{a}$.

Cependant, nous avons quelques cas où les apprenants emploient la forme des verbes de la première personne pour la troisième personne : * Carlos vais, *il ai, *elle ai. Nous trouvons aussi un cas où l'apprenant se sert du morphème de la terminaison du présent de la deuxième personne pour la première personne "*je aimes" et nous avons aussi un cas où l'apprenant utilise la forme de l'infinitif du verbe pour le présent : *je colorier.

En ce qui concerne les déterminants, nous voyons que les apprenants maîtrisent les articles définis et indéfinis et les adjectifs possessifs :

\section{Tableau 8 : Les déterminants}

\begin{tabular}{ll}
\hline \multicolumn{1}{c}{ Les articles } & \multicolumn{1}{c}{$\begin{array}{c}\text { Les adjectifs } \\
\text { possessifs }\end{array}$} \\
\hline Le chien & Mon papa \\
Le lion & Mon frère \\
Le chocolat & Mon chien \\
*Le prenom & Mon chat \\
La pizza & Mon animal \\
La plage & Ma maman \\
*La picine & Ma famille \\
*La meson & Ta maman \\
Les amis & Ton papa \\
Les lions & \\
Les animaux & \\
Les cheveux & \\
Les yeux & \\
Un restaurant & \\
*Un hotel & \\
Une maman & \\
Une famille & \\
\hline
\end{tabular}

Nonobstant, nous trouvons des cas où les apprenants se servent du déterminant de genre masculin pour déterminer les noms féminins : *le matematique, *le musique *un maison, *mon mama, *mon sour.

Par rapport à l'accord entre l'adjectif et le nom ou le pronom, nous apercevons que quelques apprenants ont pu maîtriser cette règle : mon papa est gentil, les animaux petits, mon animal préféré. Mais il y en a d'autres qui ne l'ont pas encore acquise : *la meson est petit, *deux sour, * les cheveux noire, *les yeux noire.

En ce qui concerne les prépositions, nous voyons que certaines prépositions 
apparaissent dans l'interlangue des apprenants : ${ }^{*}$ Carlos vais a la picine avec amis, *le prenom de mon chien, * ma maman travaille dans un hotel.

\subsubsection{Aspect lexical}

Par rapport à l'aspect lexical, nous trouvons que ce groupe d'apprenants a un vocabulaire qui leur permet de faire une description simple d'une personne, une présentation, parler de la famille, de quelques animaux et de quelques activités:

Tableau 9 : L'aspect lexical

\begin{tabular}{lllll}
\hline $\begin{array}{c}\text { Caractéristiques, } \\
\text { qualités et couleurs }\end{array}$ & Actions & Lieux et activités & Nourriture & Autres \\
\hline Gentil & Marcher & Piscine & Pizza & \\
Génial & Étudier & Maison & Famille \\
Petit & Chanter & Restaurant & & Maman \\
Noir & Dessiner & Hôtel & Papa \\
& Colorier & Plage & Frère \\
& Aimer & Musique & Soeur \\
& Travailler & Football & Amis \\
& Manger & Loisir & Animaux \\
& & & Chien \\
& & & Chat \\
& & & Lion \\
& & & Cheveux \\
& & & Yeux \\
\hline
\end{tabular}

Cependant, nous avons aussi des apprenants qui se servent de leur L1 pour parler de quelques caractéristiques d'êtres humains : *amoroso, *lindo, *travieso. Nous trouvons des cas où les apprenants emploient la base de leur L1 pour créer des mots de la L2 : *linde, *je tenge, *dibujer, *la montane. Nous avons aussi un cas de fauxamis avec le verbe "tenir " qui prend la fonction du verbe « avoir »: *je tian.

\subsubsection{Aspect orthographique}

En ce qui concerne l'aspect orthographique, nous voyons que la plupart des apprenants n'ont pas encore acquis les règles d'accentuation du français:
Tableau 10 : Les accents

\begin{tabular}{lll}
\hline \multicolumn{1}{c}{ Aigu } & \multicolumn{1}{c}{ Grave } & Circonflexe \\
\hline *etudie & *vais a la pi- & *hotel \\
*ecole & cine & \\
*prenom & *frere & \\
*genial & & \\
*preféré & & \\
*sinema & & \\
*electricite & & \\
\hline
\end{tabular}

Par rapport à l'élision, nous trouvons que la plupart maîtrise cette règle: elle s'appelle, mon papa s'appelle, mon frère s'appelle, je m'appelle, j'ai, j'habite. Mais nous avons encore quelques apprenants qui ne la font pas : *je aime, *je appelle. 
Nous voyons aussi des cas où les apprenants remplacent les consonnes ou les voyelles, à savoir le remplacement de la consonne " $c$ " par " $s$ " " *sine$m a "$ et le remplacement de la graphie " ai " par " $e$ " "*meson ", " oi" par " $u$ " " *mua", " au " par "o " "*otre".

Nous avons aussi des cas d'omission de la consonne « $\mathrm{s}$ » en position médiale " * picine » et de la consonne « $r$ » en position finale «*je aime joue $\varnothing$ ».

Nous trouvons aussi des cas d'omission des verbes " être " et « avoir " : *se $\varnothing$ genial, *je Ø9 ans.

Comme synthèse, nous voyons que ces apprenants ont pu intérioriser quelques règles dans leur interlangue, par exemple : la structure de base de la phrase du français, les morphèmes de la première et de la troisième personnes du singulier, l'apparition des quelques déterminants. Cependant, nous trouvons aussi des cas de surgénéralisation du déterminant masculin, l'omission de l'accord entre l'adjectif et le nom ou le pronom. Du point de vue de l'orthographe, nous pouvons dire qu'il y a surtout de l'interférence de la langue orale à la langue écrite comme les cas d'omission de consonnes ou de voyelles qui ne sont pas prononcées.

\subsection{L'analyse des productions écrites des apprenants de la sixième année}

Nous procèderont d'abord à présenter l'analyse des productions écrites de l'école República Francesa ; et puis, celle de l'école Fernando Terán Valls.

\subsubsection{República francesa}

\subsubsection{Aspect syntaxique}

En ce qui concerne l'aspect syntaxique, nous pouvons dire que la plupart des apprenants maîtrisent la règle de la structure de la phrase simple du français « sujet + verbe simple ou composé ou groupe verbal + complément " ou " sujet + verbe + attribut ", par exemple : j’ai une nouvelle, j'aime les poissons, j'adore ce voyage, j'adore cette exposition, elle adore les expositions de la mer, on aime la mer, j’ai fait des devoirs, j'ai mangé avec ma famille, *je suis allé avec ma famille a un voyage, j'aime jouer avec les dauphins, je vais faire un voyage, je pourrais aller chez vous, il fait chaud, il est très grand, *ils sont tres grands, *ma mere et mon père sont en train de dormir, il aime beaucoup la mer, *c'est genial, *c'est delicieux. Nous trouvons aussi des structures " sujet + complément + verbe » dans l'interlangue de ce groupe d'apprenants : je m'appelle..., je l'aime beaucoup, je les aime, je te propose une heure, je ne l'ai pas. Nous avons aussi quelques apprenants qui produisent des structures « sujet + verbe + complément + verbe infinitif + complément " : tu peux m'envoyer l'adresse?, tu peux me dire le lieu? Nous voyons aussi que quelques apprenants ont pu intérioriser la place des éléments négatifs dans la phrase : *je n'as pas aimé..., je n'aime pas la nuit. Nous avons aussi des structures complexes dans les productions écrites de quelques apprenants : *je veux aller avec ma soeur parce qu'elle adore les oceans, *quand nous avons nagé j'ai vu des beaucoup des poisons.

Nonobstant, nous apercevons aussi que quelques apprenants suppriment le 
sujet de la phrase : * $\varnothing$ est très jolie, * $\varnothing$ est mon prefere, *mais $\varnothing$ sera un autre jour, * $\varnothing$ sont genials. Nous avons aussi des cas où les apprenants ne mettent pas le pronom " y " qui remplace un lieu dans la phrase : *ma soeur doit $\varnothing$ aller avec moi, *ma soeur $\varnothing$ est aller aussi, *je ne peux pas $\varnothing$ aller avec ma sœur, *je dois $\varnothing$ aller avec ma sœur, *je veux $\varnothing$ venir avec ma soeur. Cela est provoqué probablement par l'interférence de la structure de la L1.

Un autre cas d'interférence de la L1 est les exemples suivants : *tu me peux donner ton adresse?, *tu me peux doner l'adresse car les apprenants ont placé le pronom complément devant le verbe conjugué pour un groupe verbal composé d'un verbe modal et d'un infinitif.

Nous trouvons aussi d'autres cas d'interférence de la L1 dans l'interlangue de ce groupe d'apprenants, par exemple : l'addition de la préposition " à » pour introduire un complément d'objet direct (*visiter a mon grant-parent) ou pour le futur proche (*aller a manger, *aller a nager), l'addition de la préposition " dans " pour introduire un complément circonstanciel de temps (*dans la tard *le soleil sortie, dans le matain), l'omission de l'adverbe " pas " pour une phrase négative (*je ne peux $\varnothing, *$ il ne va $\varnothing, * j e$ n'aime $\varnothing)$, l'omission des déterminants pour déterminer un nom (*pour $\varnothing$ enfants, *raconter $\varnothing$ autre histoire).

\subsubsection{Aspect morphologique}

Par rapport à l'aspect morphologique, nous apercevons que ce groupe d'apprenants a pu acquérir le morphème de la terminaison du présent de l'indicatif de la première et de la troisième personnes du singulier des verbes en -er et de quelques verbes irréguliers. Nous trouvons aussi les morphèmes de quelques personnes du pluriel dans l'interlangue de certains apprenants :

\section{Tableau 11 : Le présent}

\begin{tabular}{lll}
\hline \multicolumn{1}{c}{ La première personne } & \multicolumn{1}{c}{ La troisième personne } & \multicolumn{1}{c}{ Les autres personnes } \\
\hline J'aime & On nage & Tu peux \\
Je m'imagine & On aime & Nous faisons \\
Je te propose & On regarde & Nous pouvons \\
Je m'appelle & Elle adore & Nous sommes \\
*Je deteste & Elle mange & *Les daufins sont \\
*Je espere & C'est & Mon père et ma mère sont \\
Je peux & Il est & \\
Je veux & Elle est & \\
Je vais & Elle veut & \\
Je sais & Il a & \\
Je dois & Il fait & \\
Je suis & Il peut & \\
J'ai & On peut & \\
& Ma mère doit & \\
& Ma sœur va & \\
& Ma mère dit & \\
& Elle va & \\
& On va & \\
\hline
\end{tabular}


Nonobstant, nous avons aussi des cas où certains apprenants n'ont pas encore acquis les morphèmes de la terminaison du présent car ils emploient le verbe à la forme de l'infinitif, ils surgénéralisent la terminaison de la troisième personne ou la forme de la troisième personne pour les autres personnes, ils se servent de la terminaison de la première ou la deuxième personne pour la troisième personne et ils utilisent le morphème des verbes en -er pour les verbes irréguliers :

Tableau 12 : Le présent

\begin{tabular}{llll}
\hline $\begin{array}{c}\text { La terminaison de la } \\
\text { troisième personne }\end{array}$ & L'infinitif & $\begin{array}{c}\text { La terminaison de la première } \\
\text { ou la deuxième personne }\end{array}$ & $\begin{array}{c}\text { Le morphème } \\
\text { des verbes en -er }\end{array}$ \\
\hline *Je sait & $\begin{array}{c}\text { *Je savoir } \\
\text { *Je aller }\end{array}$ & $\begin{array}{l}\text { *Elle veux } \\
\text { *Le taxi peux }\end{array}$ & $\begin{array}{c}\text { *Je alle } \\
\text { *Il alle }\end{array}$ \\
*Je doit & *On manger & & \\
*Je veut & & & \\
*Je voit & & \\
*Je fait & & \\
*Je va & & \\
*Je est & & \\
*Tu est & & \\
*Tu comprendØ & & \\
*Tu peut & & \\
*Ils s'appelle & & \\
*Mon parents visite & & \\
*mes parents doit & & & \\
\hline
\end{tabular}

En ce qui concerne le passé composé, nous voyons que quelques apprenants maîtrisent sa formation et son emploi dans leurs productions : j'ai fait, j'ai mangé, j'ai vu, je suis allé, il m'a dit, il a plu, ma mère a dit, on a mangé, on a nagé, on a fait, on a $\mathrm{vu}$, mon père et ma mère ont dormi, mon frère et moi avons dessiné, nous avons nagé, nous avons fait, puisque nous avons des cas où les apprenants se servent d'un verbe en infinitif, du présent ou du participe passé pour exprimer des événements passés ; nous trouvons aussi des cas où les apprenants utilisent l'auxiliaire accompagné d'un verbe au présent ou d'un infinitif ; il existe aussi des cas d'omission de la concordance entre le sujet et le participe passé, de confusion d'auxiliaire ou d'utilisation de l'auxiliaire de la troisième personne pour les autres personnes: 
Tableau 13: Le passé composé

\begin{tabular}{|c|c|c|c|}
\hline $\begin{array}{l}\text { L'utilisation de } \\
\text { l'infinitif }\end{array}$ & $\begin{array}{c}\text { L'utilisation de } \\
\text { l'infinitif }\end{array}$ & $\begin{array}{l}\text { L'utilisation du } \\
\text { participe passé }\end{array}$ & $\begin{array}{c}\text { L'utilisation l'auxiliaire } \\
\text { accompagné d'un } \\
\text { infinitif }\end{array}$ \\
\hline *on voir & *je observe & *je parti & *on a pécher \\
\hline *on dormir & *je deteste & *je resté & *nous avons faire \\
\hline *on aller & *je nage & *je allé & *nous avons manger \\
\hline *je manger & *il reste & *je aimé & *nous avons voir \\
\hline *je voir & *on fait & *j’aimé & *nous avons nager \\
\hline \multirow[t]{6}{*}{ *je dormir } & *on peche & *j'adoré & *nous avons manger \\
\hline & *on mange & $*$ je dormi & *nous avons jouer \\
\hline & *on nage & *le soleil sorti & *mon frère et moi avons \\
\hline & *nous font & & faire \\
\hline & *mes parents se & & *Je suis aller \\
\hline & couchent & & *nous sommes monter \\
\hline $\begin{array}{c}\text { L'utilisation de } \\
\text { l'auxiliaire accom- } \\
\text { pagné d'un verbe } \\
\text { au présent }\end{array}$ & $\begin{array}{c}\text { L'omission de } \\
\text { l'accord entre le } \\
\text { pronom et le } \\
\text { participe passé }\end{array}$ & $\begin{array}{l}\text { La confusion } \\
\text { d'auxiliaire }\end{array}$ & $\begin{array}{c}\text { L'utilisation de } \\
\text { l'auxiliaire de la } \\
\text { troisième personne } \\
\text { pour les autres } \\
\text { personnes } \\
\end{array}$ \\
\hline *j'ai disfrute & *nous sommes allé & *nous avons monté & *nous a fait \\
\hline *je ai deteste & *elle est allé & \multirow{4}{*}{$\begin{array}{l}\text { *je suis aime mange } \\
\text { *je suis deteste } \\
\text { *je te suis propose } \\
\text { *la nuit a tombe }\end{array}$} & *nous ont allé \\
\hline *j'ai deteste & & & *je a fait \\
\hline *j'ai aime & & & *mon frère et moi ont \\
\hline *on a mange & & & dessiné \\
\hline \multicolumn{4}{|l|}{ *on a nage } \\
\hline \multicolumn{4}{|l|}{ *on a dessine } \\
\hline \multicolumn{4}{|l|}{ *on a joue } \\
\hline \multicolumn{4}{|l|}{ *on est tombe } \\
\hline \multicolumn{4}{|l|}{ *nous sommes } \\
\hline \multicolumn{4}{|l|}{ arribe } \\
\hline \multicolumn{4}{|l|}{ *nous avons mange } \\
\hline *nous avons nage & & & \\
\hline
\end{tabular}

Quant à l'imparfait, nous apercevons que ce temps est remplacé par le présent ou par un participe passé dans les productions écrites de ce groupe d'apprenants : *il fait chaud, *le vent est très fort, *ils sont très grands, *mon frère et moi jouons, *mes parents dormis.

Par rapport au futur proche, nous le trouvons dans la production de quelques apprenants, par exemple : je vais aller, je vais faire.
En ce qui concerne les déterminants, nous voyons que ces apprenants les emploient dans leur production, surtout les articles et les adjectifs possessifs: 
Tableau 14: Les déterminants

\begin{tabular}{|c|c|c|c|}
\hline \multicolumn{2}{|c|}{ Les articles } & \multirow{2}{*}{$\begin{array}{l}\text { Les adjectifs possessifs } \\
\text { Mon frère }\end{array}$} & \multirow{2}{*}{$\begin{array}{r}\text { Les autres } \\
\text { Ce voyage }\end{array}$} \\
\hline Le midi & Le bruit & & \\
\hline Le soleil & Le premier jour & Mon père & Ce jour \\
\hline Le climat & Le vent & Mon adresse & Cette exposition \\
\hline Le bateau & Le devoir & Ma famille & Cette année \\
\hline Le dauphin & Le nuage & Ma soeur & Ces animaux \\
\hline Le goût & Le centre & Ma mère & Tout le temps \\
\hline Le taxi & & Ma grand-mère & *Tout le paisage \\
\hline L'eau & & Mes parents & Quelle adresse \\
\hline L'adresse & & Ton invitation & Quelques heures \\
\hline L'exposition & & Ton adresse & \\
\hline L'hôpital & & Ta réponse & \\
\hline L'endroit & & Ta soeur & \\
\hline La classe & & Ta maison & \\
\hline La maison & & Ta famille & \\
\hline La mer & & Sa carte & \\
\hline La nuit & & Sa mère & \\
\hline \multicolumn{4}{|l|}{ La pluie } \\
\hline \multicolumn{4}{|l|}{ La semaine } \\
\hline \multicolumn{4}{|l|}{ Les poisons } \\
\hline \multicolumn{4}{|l|}{ Les poissons } \\
\hline \multicolumn{4}{|c|}{ Les dauphins } \\
\hline \multicolumn{4}{|l|}{ Les voyages } \\
\hline \multicolumn{4}{|l|}{ Les mers } \\
\hline \multicolumn{4}{|l|}{ Les vacances } \\
\hline \multicolumn{4}{|l|}{ Les océans } \\
\hline \multicolumn{4}{|l|}{ Les enfants } \\
\hline \multicolumn{4}{|l|}{ Les nuages } \\
\hline \multicolumn{4}{|l|}{ Un dessin } \\
\hline \multicolumn{4}{|l|}{ Un jour } \\
\hline \multicolumn{4}{|l|}{ Un salon } \\
\hline \multicolumn{4}{|l|}{ Un voyage } \\
\hline \multicolumn{4}{|l|}{ Une ile } \\
\hline \multicolumn{4}{|l|}{ Une heure } \\
\hline \multicolumn{4}{|l|}{ Une sieste } \\
\hline \multicolumn{4}{|l|}{ Une nouvelle } \\
\hline \multicolumn{4}{|l|}{ Une réunion } \\
\hline \multicolumn{4}{|l|}{ Une plage } \\
\hline \multicolumn{4}{|c|}{ Une autre heure } \\
\hline Des devoirs & & & \\
\hline Des poissons & & & \\
\hline Des fruits & & & \\
\hline Des jeux & & & \\
\hline De la natatio & & & \\
\hline De la viande & & & \\
\hline
\end{tabular}


Cependant, nous avons des cas où les apprenants se servent du déterminant masculin pour les noms de genre féminin ou du féminin pour les noms de genre féminin ; ils font l'accord selon le genre du nom de leur L1 et il y a un cas où un apprenant utilise l'adjectif possessif de genre féminin pour un nom qui commence par voyelle :

\section{Tableau 15 : Les erreurs d'utilisation des déterminants}

\begin{tabular}{|c|c|c|c|}
\hline $\begin{array}{l}\text { L'utilisation } \\
\text { du masculin }\end{array}$ & $\begin{array}{c}\text { L’utilisation du } \\
\text { féminin }\end{array}$ & $\begin{array}{l}\text { L'utilisation des déter- } \\
\text { minants selon le genre } \\
\text { de noms de la Ll }\end{array}$ & $\begin{array}{l}\text { L'utilisation de l'adjectif } \\
\text { possessif féminin pour un } \\
\text { mot de genre féminin qui } \\
\text { commence par voyelle }\end{array}$ \\
\hline $\begin{array}{l}\text { *le nuit } \\
\text { *le nourriture } \\
\text { *le chose } \\
\text { *le heure } \\
\text { *le famille } \\
\text { *le soeur } \\
\text { *le mer } \\
\text { *le mère } \\
\text { *le invitation } \\
\text { *le adresse } \\
\text { *le pluie } \\
\text { *un chose } \\
\text { *un heure } \\
\text { *ce aventure }\end{array}$ & $\begin{array}{l}\text { *une voyage } \\
\text { *une bateau } \\
\text { *une poison } \\
\text { * sa message } \\
\text { *cette voyage }\end{array}$ & $\begin{array}{l}\text { *le fin } \\
\text { *du glace } \\
\text { *la matin } \\
\text { *tous les années } \\
\text { *tous la famille } \\
\text { *tout les choses } \\
\text { *tout les deux semaines }\end{array}$ & *ta invitation \\
\hline
\end{tabular}

Quant à l'accord de l'adjectif qualificatif et du nom ou pronom, nous trouvons que la plupart des apprenants le font, par exemple : ma petite sour, le vent est très fort, ils sont très grands, la mer est très belle, des choses importantes, *c'est tres genial, c'est super, *c'est delicieux, deux semaines, trente minutes, la semaine dernière, la première semaine, la nouvelle expo. Nonobstant, nous avons des cas où les apprenants ne font pas l'accord : *l'île est très grand et joli, *deux semaines genial. Nous trouvons aussi des cas où les apprenants font l'accord avec le nom au lieu du pronom démonstratif neutre: *nous avons mange de le nourriture c'est très bonne, *ce sont très jolies. Nous voyons aussi un cas où l'apprenant emploie un adjectif de genre féminin pour qualifier un nom de genre masculin : *les daufins sont très belles. Nous avons un cas d'omission du morphème pluriel pour un nom pluriel : *les travailø.

Nous apercevons que dans l'interlangue de ce groupe d'apprenants, il y a l'utilisation de quelques pronoms toniques : mon frère et moi, avec moi, avec toi, pour moi. Mais nous avons aussi des cas où quelques apprenants emploient un pronom sujet avant la conjonction " et " au lieu d'un pronom tonique dû à l'interférence de la L1: *je et mon famille, *je et ma sœur. 
Nous trouvons aussi des cas où les apprenants accordent une nouvelle fonction au pronom relatif "qui " qui prend celle de la conjonction "que " ou du pronom "que ": *je m’imagine qui bien, *ma mère a dit qui je veux aller avec sœur, *tout les choses qui on fait.

Par rapport aux expressions de quantité, nous voyons que quelques apprenants ont intériorisé la règle car ils se servent de la préposition "de " après l'adverbe "beaucoup ": beaucoup de poisons, beaucoup de poissons. Mais nous avons encore des apprenants qui surgénéralisent les articles partitifs après une expression de quantité : *un peu de la viande, *beaucoup des choses, *beaucoup des poisons.

En ce qui concerne les prépositions, nous apercevons l'apparition de certaines dans l'interlangue de ce groupe d'apprenants, à savoir "de, dans, pour, à, avec, en, sur, chez, pendant": l'adresse de l'endroit, *je aime nager dans l'eau, *nous avons nager dans la mer, dans le bateau on fait le devoir, *nous sommes arribe a une ile, *je peux le 14 feubier à 10 heures, *a la fin mon frère et moi nous faisons un desin, *tu me peux responder ̀̀ mon adresse demain, *on va a la classe de danse, *aller a la plage, *je peux ir avec ma sœur, *je ne peux pas aller avec sœur, nous avons jouer avec des animaux, j'ai mangé avec ma famille, merci beaucoup pour l'invitation, *je suis aller a le océan pacific pour 2 semaine, un voyage en bateau, *je a fait un voyage sur bateau, *pendant la matin nous avons voir de dauphins, *pendant la nuit a tombe la pluie, *pendant mes parents dormis, mon frère et moi avons faire de dessins, je pourrais aller chez vous.

Cependant, nous voyons que la préposition "dans " prend la fonction de "pendant " ou de " à " à cause de l'interférence de la L1 : *ce que nous avons faire dans ce voyage, *dans les voyages c'est tout jolie, *dans ma vacances je alle a la plage, *dans le mois du juillet. Nous avons aussi le cas de la préposition "de " qui prend la fonction des articles partitifs : *manger de salade, *nous avons manger de poisson, *on a mangé de sandwich.

\subsubsection{Aspect lexical}

Par rapport à l'aspect lexical, nous apercevons que ce groupe d'apprenants a un lexique qui leur permet de parler des loisirs, des activités, de la famille, de la nourriture, de la ville, d'une personne :

Tableau 16 : L'aspect lexical

\begin{tabular}{lllll}
\hline $\begin{array}{c}\text { Caractéristiques, } \\
\text { couleurs }\end{array}$ & Actions & Lieux et activités & Nourriture & Autres \\
\cline { 3 - 5 } Fort & Nager & Lieu & Nourriture & Réponse \\
Joli & Jouer & Endroit & Aliment & Sieste \\
Grand & Détester & Aventure & Eau & Adresse \\
Content & Aimer & Voyage & Sandwich & Semaine \\
Terrible & Faire & Classe & Viande & Devoirs \\
Chaud & Espérer & Maison & Glace & Carte \\
Belle & Raconter & Cuisine & Riz & Dessin \\
Bonne & Manger & Salon & Fruits & Bruit \\
\hline
\end{tabular}




\begin{tabular}{llll}
\hline Délicieux & Ecrire & Océan & Soleil \\
Gracieux & Proposer & Hôpital & Pluie \\
Petit & Observer & Ecole & Nuage \\
Calme & Partir & Restaurant & Vent \\
Triste & Aller & Plage & Animaux \\
Différent & Dormir & Parc & Dauphin \\
Important & Inviter & Cinéma & Poisson \\
Super & Envoyer & Exposition & Famille \\
Fatigué & Rester & Ile & Mère \\
Blanc & Pratiquer & Natation & Père \\
Gris & Arriver & Volleyball & Soeur \\
& Changer & Danse & Frère \\
& Voir & Réunion & Grand-mère \\
& Monter & & Parents \\
& Veni & & Bateau \\
& Monter & & Taxi \\
& Venir & & Vacances \\
\hline
\end{tabular}

Nonobstant, nous voyons que quelques apprenants se servent de leur L1 dans leurs productions écrites : *la paz, *le cielo, *le sonrisa, *les mareas, *tu me le peux envier par mensaje, *proponer de ir, *responder. Nous avons aussi des cas où les apprenants utilisent un lexème de leur L1 pour créer des mots de la L2 : *la pesque, *olages, *le recorride, *une tormente, *une ensalade, *le igles, *un libre, *dormir dans le barc, *c'est aterratour, *j'ai disfrute, *atrape du poisson, *regrese a la maison, *tu peux me toper, *on converce, *on nade, *ma soeur le encant la mer, *c'est tres diverte, *envier, *mon père se levanté. Nous trouvons aussi un cas de faux-amis pour le mot "tard" qui a le sens de "après le moment voulu " en français au lieu d'utiliser le mot composé "après-mi$d i$ " qui a le sens de "tarde " en espagnol: *dans le tard.

\subsubsection{Aspect orthographique}

En ce qui concerne l'aspect orthographique, nous pouvons dire que nous avons des apprenants qui mettent les différents accents sur les mots, par exemple :

Tableau 17 : Les accents

\begin{tabular}{lll}
\hline \multicolumn{1}{c}{ Aigu } & \multicolumn{1}{c}{ Grave } & \multicolumn{1}{c}{ Circonflexe } \\
\hline Océan & Très & Bientôt \\
Resté & À 10 heures & Gô̂t \\
& Après & \\
& Frère & \\
& Père & \\
& Mère & \\
& Grand-mère & \\
\hline
\end{tabular}

Cependant, nous voyons aussi que d'autres apprenants ne mettent pas les accents sur les mots : 
Tableau 18 : L'omission des accents

\begin{tabular}{lll}
\hline \multicolumn{1}{c}{ Aigu } & \multicolumn{1}{c}{ Grave } & Circonflexe \\
\hline *reponse & *tres & *ile \\
*reunion & *apres & *hopital \\
*video & *a une ile & *ma famille \\
*ocean & *je espere & peche \\
*expose & *mon frère & *etre \\
*Amelie & prefere & \\
*genial & *premiere & \\
*egale & *quatrieme & \\
*nous & *deuxieme & \\
avons & *troisieme & \\
mange & *metre & \\
*on a & & \\
dessine & & \\
*on est & \\
tombe & \\
*je deteste & \\
*mon & \\
frère pre- & \\
fere & \\
*ecrit & \\
*delicieus & \\
*different & \\
*electro- & \\
nique & \\
\hline
\end{tabular}

Nous avons aussi des cas où les apprenants emploient l'accent aigu au lieu de l'accent grave ou circonflexe : *mére, *pére, *oú, *la péche.

Quant à l'élision, nous voyons que certains apprenants maîtrisent la règle : j'ai fait, j’ai, je m'imagine, tu peux m'envoyer, c'est, parce qu'elle ; et d'autres ne la font pas: *je espere, *je aime, *je arrive, *le océan, *le invitation, *le adresse.

Nous trouvons aussi des cas où les apprenants remplacent les consonnes ou les voyelles, à savoir le remplacement de la consonne " $v$ " par " $b$ " " *arribe, *feubier, *obserbe ", de "b" par " $v$ " "*vataux, *von", de "d"par
" $t$ " "* grant-parent ", de " $t$ " par " $d "$ " *ma soeur peud", de " $x$ " par " $s "$ " *delicieus ", de "j" par "y " *deya", de la graphie "ph" par "f" "*daufins, *daufan ", de "qu " par "c " " *acific ", de "ll" par "jou y " " mejeur, *travaiye ", de "in " par " an ou ain ou en ou am" "*dauphand, *daufan, *anvitation, *matain, *enviter, *amportance, *ambiter", de "en " par " an ou in" " *contant, *rancontre, *randez-vous, *nous sintions", de "an" par " en " "viende ", de "ou " par " $u$ " "*beacup, *puvons, *jur, *nus, *juer", de "ai " par "e " " *me après, *je ne se pa, *fer de dessin, *meson, *lesé ", de "y " par " $i$ " " *paisage, *tipes " et de "au " par " o " "*otre, *orvoir".

Nous avons aussi des cas d'omission des consonnes finales " $s$ " "*des alimentØ, *mes parentØ, *nous la faison $\emptyset$, *nous sommeØ, *tempØ , *je ne se paØ», " $r$ " "*tu peux donne $\boldsymbol{\emptyset}$ * "pour mange " $t$ " " *conten $\boldsymbol{\varnothing , ~ * c l i m a \emptyset , ~ * r e s t a u r a n ~} \boldsymbol{\emptyset}$, *finalemenØ "; des consonnes médiales "s " "*poisØons, *desØin, *desØiné", " $m$ " "*nous somØes, *comØencé ", "c" "*acØepte ", " $n$ " "*donØer, *anØés ", "p " "*apØorte ", "l" "*balØon "; de la consonne initiale " $h$ " "* Øorrible "; de la voyelle " $e$ " en position finale absolue "*anéØs, *fairØ, *pluiØ", en position initiale "*je Øspere" et en position médiale "*nous nagØons, *nous mangØons".

Nous trouvons aussi des cas d'addition de consonnes: *jollie, *addresse *maissons, *peut calme, *ont nage.

Finalement, nous avons des cas d'omission du verbe " être " : *se $\boldsymbol{\emptyset}$ tres jolie, *se Ø terrible, *se $\boldsymbol{\emptyset}$ très bien, *ce $\varnothing$ terrible.

Comme synthèse, nous apercevons que ces apprenants ont pu intérioriser quelques règles dans leur interlangue, par exemple : la structure de la phrase 
simple du français, le morphème du présent pour les verbes en -er de la première personne et de la troisième personne du singulier, l'utilisation de déterminants pour introduire un groupe nominal. Cependant, ils ont encore des règles qu'ils n'ont pas pu acquérir, à savoir : l'utilisation de la préposition " à " pour introduire un complément d'objet direct, la surgénéralisation de la terminaison de la troisième personne du singulier pour les autres personnes, les règles de formation et d'emploi du passé composé et de l'imparfait, l'interférence de la langue orale au moment d'écrire les mots dans la langue écrite.

\subsubsection{Fernando Terán Valls}

\subsubsection{Aspect syntaxique}

Quant à l'aspect syntaxique, nous apercevons que la plupart des apprenants maîtrisent la règle de la structure de la phrase simple du français " sujet + verbe simple ou composé ou groupe verbal + complément " ou "sujet + verbe + attribut ", par exemple : ma soeur est blonde, ils sont très beaux, *c'etait très amusant, *il y a un petit probleme, *elle a de la gripe, ma sœur aime la mer, *nous avons connu un dalfant, j'ai fait un voyage, j'ai fait un voyage avec ma famille, j'ai détesté les repas, on a eu peur le deuxième jour, on a vu des dauphins, nous avons nagé, nous avons joué, nous avons mangé des poissons, nous avons mangé du poisson, nous nous sommes bien amusés, *je suis allé avec ma sœur, *nous avons vu beaucoup des dauphins, je dois rester avec elle, *je dois venir avec ma sœur, *je vais aller avec ma sour. Nous avons aussi des structures "sujet + complément + verbe ou sujet + verbe modal
+ complément + infinitif» dans l'interlangue de ce groupe d'apprenants : je te propose de nos voir, je dois le soigner, tu peux me donner l'adresse de l'exposition. Nous trouvons aussi que les apprenants ont pu acquérir la règle de la place des éléments négatifs dans la phrase : je n'aime pas, nous n'avons pas peur, je ne sais pas, je n'ai pas aimé. Nous avons aussi des structures complexes dans les productions écrites de quelques apprenants, par exemple : *j'espere que tu me comprend, *c'est une famille qui est allé du viage a le mer, je me souviens que j'ai dessiné une iguane avec ma sœur, *j'espere que tu vas bien, j'ai aimé quand on est tombé du bateau, *comme nous sommes dans un bateau, on a mangé seulement les poissons qu'on a pêché, *je dois venir avec ma sœur parce qu'elle est malade.

Cependant, nous apercevons aussi des cas d'omission de sujet dans la production écrite d'un apprenant " * $\boldsymbol{\varnothing}$ pouvons retourner "; d'omission de la préposition "de " "*mes parents ont décidé $\boldsymbol{\emptyset}$ aller, *mon pére a decidé $\boldsymbol{\emptyset}$ pecher, *beaucoup $\varnothing$ poissons ". Ces phénomènes sont provoqués probablement par l'interférence de la L1.

Nous avons aussi un cas d'addition de l'article "des": *nous avons vu plusieurs des choses. Nous apercevons aussi des cas d'addition de la préposition " dans " à cause de l'interférence de la L1 : *dans la nuit nous sommes dormi, *dans les matin nous avons nage. Nous trouvons un cas d'interférence de la L1 pour la place de l'adverbe dans la phrase : *nous nous sommes amusés beaucoup car l'apprenant devrait mettre l'adverbe "beaucoup " après le verbe auxiliaire au lieu de le placer après le participe passé. 


\subsubsection{Aspect morphologique}

En ce qui concerne l'aspect morphologique, nous pouvons dire que ces apprenants ont pu intérioriser le morphème de la terminaison du présent de l'indicatif de la première de la deuxième et de la troisième personnes du singulier des verbes en -er et de quelques verbes irréguliers. Nous trouvons aussi les morphèmes de quelques personnes du pluriel dans l'interlangue de certains apprenants :

Tableau 19 : Le présent

\begin{tabular}{lll}
\hline \multicolumn{1}{c}{ La première personne } & \multicolumn{1}{c}{ La troisième personne } & \multicolumn{1}{c}{ Les autres personnes } \\
\hline Je te donne & Ma sœur aime & Tu tournes \\
Je te propose & Elle aime & Tu passes \\
Je vous demande & C'est & Tu me comprends \\
Je n'aime pas & Elle est & Tu vas \\
J'aime & Elle peut & Tu veux \\
J'habite & Elle dit & Tu peux \\
J'imagine & Ma mère veut & Nous pouvons \\
J'adore & Elle a & Nous sommes \\
Je déteste & Il a & Ils doivent \\
Je retourne & Il y a & Mes parents vont \\
Je te remercie & & \\
*j'espere & & \\
Je me souviens & & \\
Je suis & & \\
Je veux & & \\
Je ne peux pas & & \\
Je dois & & \\
Je vais & & \\
Je ne sais pas & & \\
\hline
\end{tabular}

Cependant, nous avons aussi des cas où certains apprenants n'ont pas encore intériorisé les morphèmes de la terminaison du présent, puisqu'ils utilisent le verbe à la forme de l'infinitif, ils surgénéralisent la terminaison de la troisième personne ou la forme de la troisième personne pour les autres personnes et ils se servent de la terminaison de la première ou la deuxième personne pour la troisième personne, par exemple: *je te dire, *elle me dire, *ma maman travailler, *la mer ne être pas, *les parents s'andormir, *je peut, *je veut, *je dit, *je sait, *je suit, *je $\boldsymbol{v a},{ }^{*} j e \boldsymbol{e s t},{ }^{*} j e \boldsymbol{a},{ }^{*} t u$ me comprend $\boldsymbol{\varnothing}$, *tu m'invite, *nous nage, *nous joue, * ma sœur veux.

Par rapport au passé composé, nous apercevons que quelques apprenants maîtrisent sa formation et son emploi dans leurs productions : je n'ai pas aimé, j'ai fait, j'ai aimé, j'ai dessiné, j'ai détesté, j'ai écrit, j'ai reçu, ce voyage a été, le jour a été, ma mère a $\boldsymbol{v} \boldsymbol{u}$, on $\boldsymbol{a} \boldsymbol{e u}$, on $\boldsymbol{a} \boldsymbol{v} \boldsymbol{u}$, on $\boldsymbol{a}$ mangé, on $\boldsymbol{a}$ pêché, nous avons connu, nous avons vu, nous avons fait, nous avons nagé, nous avons mangé, nous avons joué, nous avons regardé, *nous avons 
ecrit, nous avons regardé, *mes parents ont decidé, ces vacances ont été, je suis allé, on est tombé, ma famille et moi sommes allés, nous sommes partis, nous nous sommes bien amusés.

Cependant, nous trouvons des cas où les apprenants se servent d'un verbe en infinitif, du présent ou du participe passé pour exprimer des événements passés ; nous trouvons aussi des cas où les apprenants emploient l'auxiliaire accompagné d'un verbe au présent ou d'un infinitif ; il existe aussi un cas d'omission de la concordance entre le sujet et le participe passé, de confusion d'auxiliaire ou d'addition de l'accord entre le sujet et le participe passé avec le verbe « avoir »:

Tableau 20: Le passé composé

\begin{tabular}{|c|c|c|c|c|c|}
\hline $\begin{array}{c}\text { L'utilisation de } \\
\text { l'infinitif }\end{array}$ & $\begin{array}{c}\text { L'utilisation du } \\
\text { présent }\end{array}$ & \multicolumn{3}{|c|}{$\begin{array}{l}\text { L'utilisation du } \\
\text { participe passé }\end{array}$} & $\begin{array}{c}\text { L'utilisation de } \\
\text { 'auxiliaire accompagné } \\
\text { d'un infinitif }\end{array}$ \\
\hline $\begin{array}{l}\text { *mes parents dormir } \\
\text { *on ne faire pas }\end{array}$ & $\begin{array}{l}\text { *un famille fait } \\
\text { *elle me dit } \\
\text { *elle aime } \\
\text { *j'aime } \\
\text { *je nage } \\
\text { *nous pechons }\end{array}$ & \multicolumn{3}{|c|}{$\begin{array}{l}\text { *la famille na } \\
\text { *la famille all } \\
\text { *ils nagé } \\
\text { *je pensé } \\
\text { *je nagé } \\
\text { *je fait } \\
\text { *nous nagé } \\
\text { *nous mangé }\end{array}$} & $\begin{array}{l}\text { nous avons dormir } \\
\text { nous avons voir }\end{array}$ \\
\hline $\begin{array}{c}\text { L'utilisation de } \\
\text { l'auxiliaire accompagné } \\
\text { d'un verbe au présent }\end{array}$ & $\begin{array}{r}\text { L'omission } \\
\text { Ié } \quad \begin{array}{c}\text { l'accord entre } \\
\text { et le participe }\end{array}\end{array}$ & & $\begin{array}{r}\text { La co } \\
\text { d'aux }\end{array}$ & $\begin{array}{l}\text { usion } \\
\text { iaire }\end{array}$ & $\begin{array}{c}\text { L'addition de l'accord } \\
\text { entre le pronom et le } \\
\text { participe passé avec } \\
\text { le verbe « avoir » }\end{array}$ \\
\hline $\begin{array}{l}\text { *j'ai joue } \\
\text { *j'ai aime } \\
\text { *nous avons nage } \\
\text { *ils ont peche } \\
\text { *je suis alle }\end{array}$ & $\begin{array}{l}\text { *c'est une famill } \\
\text { alléØ }\end{array}$ & & $\begin{array}{l}\text { *ils son } \\
\text { *je suis } \\
\text { *je suis } \\
\text { *je suis } \\
\text { *je suis } \\
\text { *je suis } \\
\text { *nous } \\
\text { dormi } \\
\text { dormi } \\
\text { *nous } \\
\text { perdu } \\
\text { *nous } \\
\text { mangé } \\
\text { *nous } \\
\text { pêché }\end{array}$ & $\begin{array}{l}\text { mange } \\
\text { lage } \\
\text { mange } \\
\text { léteste } \\
\text { vu } \\
\text { aimé } \\
\text { sommes } \\
\text { sommes } \\
\text { sommes } \\
\text { sommes }\end{array}$ & $\begin{array}{l}\text { *nous avons mangés } \\
\text { *vous avez nagés } \\
\text { *vous avez pechés } \\
\text { *nous avons joués }\end{array}$ \\
\hline
\end{tabular}




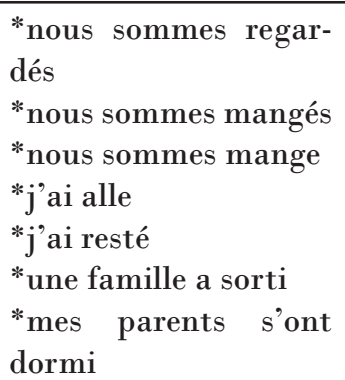

En ce qui concerne l'imparfait, nous voyons que ce temps apparaît dans la production écrite de deux apprenants, surtout avec le verbe " être ": *c'etait très amusant, tout était bien. Mais pour les autres apprenants, il est remplacé par le présent, l'infinitif ou le participe passé : *ils sont perdu, *ils sont très beaux, *elle joue, *notres parents se lever, *elle été tres contente.
Nous trouvons aussi les formes du futur proche et du futur simple dans l'interlangue de quelques apprenants pour parler de l'avenir : *ma mere va aller, il sera, ça me donnera.

Par rapport aux déterminants, nous pourrons dire que ces apprenants les emploient dans leur production, surtout les articles et les adjectifs possessifs :

\section{Tableau 21: Les déterminants}

\begin{tabular}{lll}
\hline \multicolumn{1}{c}{ Les articles } & \multicolumn{1}{c}{ Les adjectifs possessifs } & \multicolumn{1}{c}{ Les autres } \\
\hline Le voyage & Ma mère & Ce jour \\
Le bateau & Ma soeur & Cette exposition \\
*Le deuxieme jour & Ma famille & Ces vacances \\
Le dernier jour & Ma grand-mère & Tout le voyage \\
Le goût & Ma tante & Toute la mer \\
Le bar & Ma chambre & \\
Le vélo & Mon père & \\
Le message & Mon adresse & \\
Le café & Mes parents & \\
Le soir & Mes frères & \\
Le devoir & Mes vacances & \\
L'invitation & Mes devoirs & \\
L'exposition & Ton invitation & \\
L'école & Ta réponse & \\
L'enfant & & \\
L'adresse & & \\
L'heure & & \\
L'environnement & & \\
La viande & & \\
La nuit & & \\
La famille & & \\
& & \\
\hline
\end{tabular}


Nonobstant, nous avons des apprenants qui se servent de l'article ou de l'adjectif masculin pour déterminer des noms de genre féminin, à savoir "*le mer, *le information, *le plage, *le chosse, *le exposition, *le semaine, *un famille, *un histoir, *un idée, *un chatte, *un question, *au discothèque, *son fille, *ton reponse, *du mer, *quel est l'adresse, *ce heure, *cet exposition, *tout le semaine, *tout ma famille ". Nous trouvons aussi des cas où les apprenants emploient les déterminants féminins pour les noms de genre masculin "*une bar, *sa jour, *cette voyage, *cette titre, *une voyage, *une batiment ". Nous apercevons aussi des cas d'interférence où les apprenants utilisent les déterminants selon le genre du nom de leur L1, par exemple : *une matin, *la matin, *toutes les devoires. Nous avons aussi des cas où les apprenants surgénéralisent l'adjectif possessif de genre féminin pour déterminer des noms féminins qui commencent par voyelle, à savoir "*ta invitation, *sa exposition ". Finalement, nous trouvons un cas où l'apprenant surgénéralise le morphème du pluriel -s pour l'adjectif possessif de la première personne du pluriel : *notres parents.

Par rapport à l'accord entre l'adjectif et le nom ou le pronom, nous pouvons dire que quelques apprenants le font, par exemple : ils sont très beaux et grands, ils sont très beaux, c'était très amusant, les animaux aquatiques, des baleines très belles, un bon exposé, ma sour est blonde, une exposition très intéressante, ces vacances ont été très amusantes. Cependant, nous avons des cas où les apprenants ne font pas l'accord : *la semaine dernier, *les vacances dernier, *il a les yeux bleu, *elle est très content, *le mer est très grand, *mon père et ma mère sont très fatigué.
En ce qui concerne les pronoms toniques, nous voyons que les apprenants ont pu acquérir les règles : $m a$ famille et moi, avec toi.

Quant aux expressions de quantité, nous avons des cas où les apprenants emploient la préposition " de " après celles-ci : beaucoup de détail ; mais nous trouvons des cas où les apprenants surgénéralisent les articles partitifs après l'expression de quantité : *beaucoup des dauphins, *beaucoup des poissons.

Nous avons un cas d'omission de la contraction entre la préposition et l'article : *de le 15 septembre.

Par rapport aux prépositions, nous apercevons l'apparition de certaines dans l'interlangue de ce groupe d'apprenants, à savoir "de, dans, pour, à, avec, en, sur, chez, pendant, devant ": vous avez nagé dans la mer, merci beaucoup pour l'invitation, pour moi ces vacances ont été très amusantes, je fais un voyage sur un bateau, *nous avons fait voyage en bateau pendant deux semaines, j'ai détesté faire mes devoirs pendant mes vacances, nous avons nagé pendant une heure, chez ma tante, chez toi, j'ai dessiné une iguane avec ma sœur, *je peut aller avec toi, *tu tournes a gauche, *tu passes devant l'internet, les parents de Jacob, l'anniversaire de Jacob. Cependant, nous trouvons quelques prépositions qui prennent la fonction d'une autre préposition ou d'un article, à savoir la préposition "dans " acquiert la fonction de "pendant" pour les structures suivantes : *dans le jour, il peut, *dans les vacances derniers ma famille et moi sommes allés a le plage; et la préposition "de " prend la fonction de l'article « du » pour la structure suivante: *nous mangé de poisson. 


\subsubsection{Aspect lexical}

En ce qui concerne l'aspect lexical, nous voyons que ce groupe d'apprenants a un lexique qui leur permet de parler des loisirs, des activités, de la famille, de la nourriture, de la ville, d'une personne :

Tableau 22: Aspect lexical

\begin{tabular}{|c|c|c|c|c|}
\hline Caractéristiques, couleurs & Actions & Lieux et activités & Nourriture & Autres \\
\hline Amusant & Aimer & Mer & Poissons & Voyage \\
\hline Aquatique & Manger & Océan & Viande & Carnaval \\
\hline Joli & Nager & Exposition & Pain & Anniversaire \\
\hline Triste & Dormir & École & Riz & Devoir \\
\hline Bon & Pêcher & Plage & Salade & Message \\
\hline Horrible & Aller & Bar & Café & Réponse \\
\hline Simple & Dire & Maison & Glace & Dessin \\
\hline Petit & Voir & Hôpital & & Problème \\
\hline Grand & Dessiner & Discothèque & & Vacances \\
\hline Gracieux & Se souvenir & Chambre & & Adresse \\
\hline Aimable & Perdre & & & Bateau \\
\hline Malade & Jouer & & & Vélo \\
\hline Content & Dîner & & & Jour \\
\hline Intéressant & Écrire & & & Semaine \\
\hline Inoubliable & Donner & & & Invitation \\
\hline Principal & Sortir & & & Information \\
\hline Premier & Penser & & & Exposé \\
\hline Dernier & Comprendre & & & Famille \\
\hline Belle & Espérer & & & Ami \\
\hline Beau & Réunir & & & Enfants \\
\hline Fort & Inviter & & & Mère \\
\hline Blond & Dire & & & Père \\
\hline Drôle & Venir & & & Soeur \\
\hline Blanc & Proposer & & & Frère \\
\hline Bleu & Commencer & & & Tante \\
\hline \multirow[t]{14}{*}{ Noir } & Détester & & & Fille \\
\hline & Savoir & & & Fils \\
\hline & Regarder & & & Parents \\
\hline & Rester & & & Animaux \\
\hline & Ranger & & & Dauphins \\
\hline & Raconter & & & Iguane \\
\hline & Garder & & & Poissons \\
\hline & Tourner & & & Requins \\
\hline & Prendre & & & Chien \\
\hline & Passer & & & Chat \\
\hline & Accompag- & & & Poule \\
\hline & ner & & & Lapin \\
\hline & Soigner & & & Baleine \\
\hline & Travailler & & & Yeux \\
\hline
\end{tabular}




\begin{tabular}{ll}
\hline Toucher & Cheveu \\
Se lever & Marée \\
S'amuser & Ordinateur \\
\hline
\end{tabular}

Nonobstant, nous avons des apprenants qui se servent du lexème de leur L1 pour créer des mots de la L2 : *ils sont disfruté, *les parents a descansé, *je compre, *la toner se desvie, *le lague, *une tormet. Nous trouvons un cas où un apprenant emploie le mot de sa L1 : *la confite.

\subsubsection{Aspect orthographique}

Par rapport à l'aspect orthographique, nous pouvons dire que nous avons des apprenants qui mettent les différents accents sur les mots, par exemple :

Tableau 23 : Les accents

\begin{tabular}{lll}
\hline \multicolumn{1}{c}{ Aigu } & \multicolumn{1}{c}{ Grave } & Circonflexe \\
\hline Aimé & Très & Goût \\
Été & Première & Être \\
Allé & À & Plaît \\
Nagé & Mère & Bientôt \\
Dessiné & Père & \\
Mangé & Discothèque & \\
Exposé & & \\
C'était & & \\
Océan & & \\
École & & \\
Réponse & & \\
Marée & & \\
\hline
\end{tabular}

Cependant, nous trouvons des cas où il y a l'omission des accents sur les mots :
Tableau 24: L'omission des accents

\begin{tabular}{|c|c|c|}
\hline Aigu & Grave & Circonflexe \\
\hline *c'etait & *troisieme & *pechés \\
\hline *ecrit & *cinquieme & $*$ fache \\
\hline *reunir & *deuxieme & $*$ drole \\
\hline *interessant & $*$ tres & *batiment \\
\hline *alle & *apres & \\
\hline *deteste & *mere & \\
\hline *ecole & *pere & \\
\hline *expose & *frere & \\
\hline * genial & * grand-mere & \\
\hline \multirow[t]{4}{*}{ *video } & $*$ a $2 \mathrm{~h} 30$ & \\
\hline & *je t’espere & \\
\hline & *probleme & \\
\hline & $* a$ & \\
\hline
\end{tabular}

Nous avons aussi des cas de remplacement d'accent, à savoir l'utilisation de l'accent aigu au lieu de l'accent grave: *pére, *mére, *frére.

Quant à l'élision, nous voyons que la plupart des apprenants maîtrisent la règle: je n'ai pas aimé, j'ai, j'habite, c'était, c'est, s'appelle, qu'elle, l'adresse. Mais nous avons quelques cas où les apprenants n'ont pas appliqué la règle : *la invitation, *la exposition.

Nous avons aussi des cas de remplacement de consonnes ou de voyelles, à savoir le remplacement de la consonne " $q$ " par "c" "catrieme, *cuand, *can", de "s" par " $z$ " “"utilizes", de " $t$ " par " $d$ " "contend", de la graphie "ph" par "f" “* dofin", de "eau” ou "au” par "o" "“bato, *dofin, *dophins, *bocoup", de "ou” par " $u$ " "bocu, *nus", de "en" par "an" "*ansamble, *s'andormir, *atandu, *contante" et de "ai" par " $e$ " "*je ve".

Nous trouvons des cas d'omission des consonnes finales "s" " $s$ des dau-

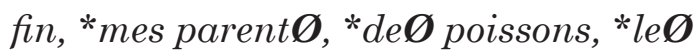


animaux, *paØ, *trèØ, *je doiØ”, "r" "pour m'inviteØ, *pour donneØ, *de t’accompagneØ, *vont alleØ, *je dois porteØ”, " $t$ ”“*ils m'onØ dit”, " $p$ "“*troØ”; des consonnes médiales " $n$ " "“conØais, *donØera, *tonØer”, “s” “"desØiné, *interesØant", " $m$ " “comØencer", " $p$ " "gripØe”, " $t$ " “未atØandu”, "l” “*tranquilØement"; de la consonne " $h$ " en position initiale " Øorrible”; de la voyelle " $e$ " en position finale absolue et position

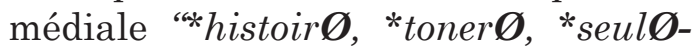
ment" et de la voyelle "u" "fatigØé".

Nous trouvons aussi des cas d'addition de consonnes ou de voyelles : *chosse, *amussent, *devoires, *aller à la mere.

Comme synthèse, nous pouvons dire que ces apprenants ont pu intérioriser quelques règles dans leur interlangue, par exemple : la structure de la phrase simple du français, le morphème du présent pour les verbes en -er de la première personne et de la troisième personne du singulier, l'utilisation de déterminants pour introduire un groupe nominal. Nonobstant, ils ont encore des règles qu'ils n'ont pas pu acquérir, à savoir : la surgénéralisation de la terminaison de la troisième personne du singulier pour les autres personnes, les règles de formation et d'emploi du passé composé, l'interférence de la langue orale au moment d'écrire les mots dans la langue écrite.

\section{Conclusions}

Comme conclusions de cet article, nous pouvons apercevoir que l'interlangue des apprenants de la troisième année de ces deux écoles est encore très instable car nous avons des phénomènes de simplification de la L2, par exemple : l'utilisation des structures simples, l'omission des déterminants, l'omission de l'accord entre l'adjectif et le nom ou le pronom ; de surgénéralisation, à savoir l'utilisation des déterminants masculins pour les noms de genre féminin ; d'interférence de la langue orale à la langue écrite comme les cas d'omission de consonnes ou de voyelles qui ne sont pas prononcées à l'oral et les apprenants ne les écrivent pas dans leurs productions écrites.

En ce qui concerne l'interlangue des apprenants de la sixième année, nous pouvons dire que c'est plus complexe que celle de la troisième année, puisque du point de vue syntaxique, nous trouvons des phrases simples avec des verbes au temps composé ou de groupe verbal composé d'un modal et d'un infinitif, il y a aussi l'apparition des structures complexes avec des pronoms relatifs ou des conjonctions subordonnées, nous avons des phrases avec des pronoms compléments ; du point de vue morphologique, nous voyons que les apprenants commencent à intérioriser les morphèmes du présent, surtout de la première et de la troisième personnes du singulier, nous trouvons aussi l'utilisation de différents déterminants pour introduire un groupe nominal, il y a l'utilisation d'une plus grande quantité de prépositions ; et du point de vue lexicale, nous apercevons une grande variété de vocabulaire qui leur permet de parler d'eux-mêmes, de la famille, d'un lieu, de goûts et de préférences.

Cependant, nous voyons qu'ils ont encore des règles qu'ils n'ont pas acquises, par exemple : nous trouvons des cas d'omission du pronom sujet dans la phrase, du pronom-adverbe « y » lorsqu'il remplace un complément circonstanciel de lieu ; l'addition de la préposition « à " pour introduire 
un complément d'objet direct ou pour le futur proche, "dans " pour introduire un complément circonstanciel de temps ; l'utilisation de la terminaison ou de la forme de la troisième personne du singulier du présent pour les autres personnes ; l'instabilité des temps passés ; l'interférence de la langue orale à la langue écrite du point de vue de l'orthographe.

\section{Bibliographie}

Alexopoulou, A. (2010). "La función de la interlingua en el aprendizaje de lenguas extranjeras". Revista Nebrija de Lingüistica Aplicada, 9, pp. 1-8.

Azzam-Hannachi, R. (2005). Evolution de l'enseignement des langues vivantes à l'école primaire en France: formation et representations des enseignants du premier degré. Thèse de doctorat: Université Nancy 2.

Brou-Diallo, C. (2007). "Interlangue ou interférence et enseignement du français langue étrangère". Revue électronique international de sciences du langage sudlangues, 7, pp. 1-15.

Corder, S.P. (1967). "The significance of learners'errors". International Review of Applied Linguistics, 5 (4), pp. $162-167$.

Corder, S.P., Bailly, D., Frauenfelder, U., Perdue, C. et Porquier, R. (1980). « La sollicitation de données d'interlangue ». Langages, 57, pp. 29-38.

Fonseca, A. (2007). "Análisis de la interlengua fónica”. Phonica, 3, pp. 3-29.
Galligani, S. (2003). "Réflexion autour du concept d'interlangue pour décrire des variétés non natives avancées en français." Revue des linguistes de l'université Paris Ouest Nanterre La Défense, 49, pp. 141-152.

Khelef, Hanane (2008). Étude morphosyntaxique des constructions prépositionnelles. Cas des écrits des étudiants de 4ème année français de l'université de Ouargla. Mémoire pour l'obtention de Magister: Université Kasdi Merbah Ouargla.

Lindqvist, C. (2006). L’influence translinguistique dans l'interlangue française: étude de la production orale d'apprenants plurilingües. Thèse pour le doctorat: Université de Stockholm.

Monville-Burston, M. et Kounouni, M. (2010). "Deux procédures expérimentales pour tester la relativisation dans l'interlangue d'apprenants chypriotes hellénophones en FLE". Advances in research on language acquisition and teaching. Selected papers. Salonique: Association Grecque de Linguistique Appliquée.

Selinker, L. (1972). "Interlanguage". International Review of Applied Linguistics, 10 (2), pp. $209-231$.

Torras, M.R. (1994). "La interlengua en los primeros estadios de aprendizaje de una lengua extranjera inglés". Comunicación, Lenguaje y Educación, 24, pp. 49-62.

Yun, H. (2009). Échanges à distance entre apprenants de FLE: étude des intéractions synchrones en contexte académique. Thèse de doctorat: Université Paris 3 - Sorbonne Nouvelle. 
